4 Bauer, H., Demerec, M., and Kaufmann, B. P., Genetics, 23, 610-630 (1938).

Bauer, H., Chromosoma, 1, 343-390 (1939).

5 Demerec, M., Cold Spring Harbor Symposia, 2, 110-115 (1934).

${ }^{6}$ Sax, K., Genetics, 25, 41-68 (1940).

\title{
ANOTHER CASE OF UNEQUAL CROSSING-OVER IN DROSOPHILA MELANOGASTER
}

By E. B. Lewis

WM. G. KeRckhoff Laboratories of the Biological Sciences, California Institute of Technology

Communicated November 18, 1940

Females homozygous for the sex-linked dominant, Bar, occasionally give rise to wild-type reversions and to forms with more extreme eye reduction than Bar. This behavior was shown by Sturtevant ${ }^{1}$ to result from unequal crossing-over. The Bar-reverted type was considered to be a deficiency for the Bar gene; while the extreme form, called Ultra-Bar or Double-Bar, was interpreted as a duplication for that gene. Later, Wright ${ }^{2}$ suggested that Bar itself had something additional present which when lost by unequal crossing-over would give back a normal chromosome (Bar-reverted).

The cytological nature of Bar was cleared up independently by Bridges ${ }^{3}$ and Muller, et al., ${ }^{4}$ who investigated the salivary gland chromosomes. They found Bar to be a tandem duplication, in normal order, for an $X$ chromosome section composed, according to Bridges' detailed analysis, of six bands. Bridges further demonstrated that Bar-reverted had the identical banding of a normal chromosome, whereas Double-Bar had a serial triplication for the region present twice in Bar and once in Barreverted.

The second case of a tandem duplication being responsible for a dominant "mutation" is that of the sex-linked Hairy wing, which Demerec ${ }^{5}$ has shown is a repetition for a single heavy band near the tip of the $X$ chromosome. However, its location in a region of extremely low crossingover prevented a study of unequal crossing-over.

This paper is a preliminary report on an autosomal tandem repeat which was detected as a suppressor of the dominant mutant, Star $(S, 2$ 1.3).

An analysis of the salivary gland chromosomes of this suppressing factor, when homozygous, when closely paired with a normal chromosome and when present as an unpaired haploid strand, consistently showed the presence of a tandem duplication in direct order near the left end of the 
second chromosome. The section present twice appears to include the two faint bands, $21 D 4-5$, and the heavy, frequently capsulated doublet, $21 E 1-2$; i.e., a section of at least four bands.

$D p(2) S$ appeared in a study of changes at the Star locus, a consideration of which is essential before discussing the properties of this repeat. $S /+$ has roughened, slightly reduced eyes; $S / S$ is always lethal. Star-recessive $\left(S^{r}\right)$ is the name tentatively given to a recessive mutant near if not at the Star locus. $S^{\gamma} / S^{\gamma}$ has smaller, rougher eyes than $S /+$, and may have gaps at the tips of the wing veins. The compound, $S / S^{\prime}$, is much more extreme, having a narrow diamond-shaped eye and extensively interrupted venation.

Bridges has reported that the salivary gland chromosomes of Star are apparently normal. The same appears to be true for Star-recessive and also for the dominant Suppressor of Star $(S u-S, 2-1.3 \pm)$ found by Curry.

The allelic relation between $S$ and $S^{r}$ is, as yet, ambiguous. From al $S$ ho $/ S^{\prime}$ females $(a l=$ aristaless, $2-0.0 ;$ ho $=$ heldout, $2-4.0)$, wild-type "reversions," which are always heldout crossovers and which are cytologically normal, occur with a frequency of $0.01 \%(4: 31,106)$; by using females heterozygous for inversions in all of the other chromosome arms, their frequency has been stepped up to $0.046 \%(12: 26,370)$. Yet, a crossover complementary to the reversions has not been detected. The situation may be similar to a case, recently reported by Oliver, ${ }^{6}$ of reversions, associated with crossing-over in one direction, arising from females carrying two alleles of the lozenge eye mutation. For the sake of simplicity, $S$ and $S^{r}$ are considered as alleles in this paper.

$D p(2) S$ arose spontaneously as a single individual among approximately 49,000 offspring of al $S^{\gamma}$ ho/ $S^{\gamma}$ females individually mated to al $S$ ho/Cy, $E-S$ males. The fly had normal aristae, nearly wild-type (Star suppressed) eyes and heldout wings. Tests showed that the mother had contributed $a l^{+}, h o$ and $D p(2) S$, whose origin was therefore associated with crossingover. $\quad D p(2) S /+$ and $D p(2) S / S^{\psi}$ look wild-type. $\quad D p(2) S / D p(2) S$ is also normal except for an occasional slight extra vein near the fifth longitudinal vein. This wild-type action is in striking contrast to the pronounced phenotypic effects of Bar and Hairy wing.

A study of unequal crossing-over in the heterozygous duplication has shown that the Star locus is included in the repeated sections; i.e., it has been possible to recover from al $D p(2) S h o / S$ females unequal crossover products which have $S$ inserted into the left (distal) section of the repeat, and others with $S$ introduced into the right (proximal) region. The latter occur with a frequency of $0.3 \%(6: 1948)$ or roughly thirty times as frequently as the former $(0.01 \%$ or $2: c a 23,000)$. In terms of genetic length this indicates that the $S$ locus is included in the extreme right portion of 
each of the two regions present in duplicate. The original duplication, since it arose from homozygous $S^{\prime}$, might be expected to have a $S^{\prime}$ gene in each of these positions. Using parentheses to bound the repeated regions, its composition may be written: $D p(2) S=\left(\ldots S^{r}.\right)\left(\ldots S^{r}.\right)$. That $S^{r}$ is present in the proximal section has been demonstrated by its recovery from

$$
\frac{a l\left(\ldots S^{r} .\right)\left(\ldots S^{r} .\right) \quad h o}{(\ldots S .)}
$$

females as $S^{\gamma}$ ho cross-overs, whose cytological picture is normal; their frequency is $0.35 \%(57: 16,568)$ or approximately that of the complementary $a l\left(\ldots S^{\prime}.\right)(\ldots S$.$) class mentioned above. In the two cases where S$ was inserted into the left section, the product may be written: (...S.)$\left(\ldots S^{r}\right.$.) ho, and its origin visualized as the result of the following pairing:

$$
\frac{a l\left(\ldots S^{r} .\right)\left(\ldots S^{r} .\right) \text { ho }}{(\ldots S .)},
$$

accompanied by a cross-over between the $S$ locus and the break point of the duplication. The complementary crossover is expected in this case to be $a l S^{r}$, or the removal of $S^{r}$ from the left section. Yet, although a total of $243 S^{r}$ ho types have been detected, no cases of al $S^{r}$ have occurred. This may mean that $S^{r}$ is slightly to the right of $S$, as was suggested, in part, by evidence given above. On this basis, either $S^{r}$ is just outside the duplication or it is so close to the break point that crossingover has failed so far to remove it from the left section.

Although the phenotypic effects of the original duplication are consistent with the assumption that one $S^{\gamma}$ and a normal allele of $S^{\gamma}$ are acting, the origin of $D p(2) S$ from homozygous $S^{\gamma}$ would seem to indicate that this action is more likely a position effect. A preferable notation, for the present, would be $D p(2) S=\left(\ldots S^{r}\right.$ ?. $)\left(\ldots S^{r}.\right)$.

From al (...S ?.)(...S $S^{r}$.) ho/ + females, normal $S^{r}$ ho chromosomes and $a l\left(\ldots S^{r}\right.$ ?. $)\left(\ldots S^{r+}\right.$.) occur with approximately equal frequencies as expected.

There is genetic evidence, not of a crucial character, that the locus of net $(2-0.3 \pm)$ is also included in $D p(2) S$ at the extreme left end of each section. If this is the case then the total frequency of crossing-over between the loci of net and Star in heterozygous $D p(2) S$ is greater when the distal section is involved $(1.4 \%)$ than when the proximal one takes part $(0.7 \%)$. As in the experiments previously given, these data are obtained from females heterozygous for inversions in some of the other chromosome arms with the result that the normal net-S distance of $1 \%$ is increased to $2 \%$ or more. 
From females homozygous for the original duplication whose repeated sections may be supposed occasionally to pair in an unequal manner, diagrammatically,

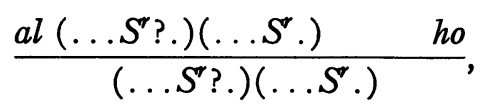

two types of unequal cross-over products have been obtained which, apart from phenotype, are analogous to the derivatives produced by homozygous Bar females. The normal chromosome products, corresponding to Bar-reverted, are detected on the basis that they carry $S^{r}$; their frequency is $0.25 \%$ (9al $\left.S^{\gamma}+5 S^{\gamma} h o: 5594\right)$. New chromosomes with three sections in tandem repetition, as is the case with Double-Bar, occur with approximately the same frequency as the normal types, namely, $0.18 \%$ $(8 a l+3 h o$, triplications:6000); their action is to suppress, completely, $S E-S(E-S=$ Enhancer of Star, 2- 6. $)$, whereas $D p(2) S$ (..S ?.) $\left(\ldots S^{\prime}\right.$.) only partially suppresses the small rough eye effect seen in $S$ $E-S /+$. The unequal crossover types have been examined in the salivary gland chromosomes and the analogy with the Bar derivatives has been found to hold. The homozygous triplication, symbol, $\operatorname{Tr}(2) S$, has slightly bulging eyes with large facets; in addition to occasional slight extra veins, described for the homozygous $D p(2) S$, there is often a branching of the second longitudinal vein. The wing effects are perhaps to be ascribed to the locus of net.

Homozygous $\operatorname{Tr}(2) S$ females have produced $S^{\prime \prime}$ chromosomes with only one section present, and also a new "dominant" unequal crossover product, which over $S^{r}$ (or $S^{r+}$ ) has eyes resembling those of homozygous $T r$ (2)S. A cytological analysis supports the conclusion that the "dominant" is a repeat of five sections. When homozygous this quintuplication, symbol, $Q n(2) S$, is still quite viable and fertile, and has the same effects, but much more intensified, as homozygous $\operatorname{Tr}(2) S$. $Q n(2) S$ would correspond to Quadruple-Bar obtained by Rapoport ${ }^{7}$ from attached- $X$ females homozygous for Double-Bar.

Summary.-1. An autosomal tandem duplication is described, whose origin was associated with crossing-over.

2. Genetic evidence indicates that the locus of Star and possibly the net locus are included in each of the duplicate sections.

3. The homozygous duplication gives unequal crossover products analogous to Bar-reverted and Double-Bar. A repeat of five sections has been derived from the homozygous triplication.

${ }^{1}$ Sturtevant, A. H., Genetics, 10, 117-147 (1925).

2 Wright, S., Amer. Nat., 63, 479-480 (1929).

3 Bridges, C. B., Science, 83, 210-211 (1936). 
4 Muller, et al., C. R. Acad. Sc. USSR, 1 (X), 87-88 (1936).

- Demerec, M., Genetics, 24, 271-277 (1939).

- Oliver, C. P., Proc. Nat. Acad. Sci., 26, 452-454 (1940).

${ }^{7}$ Rapoport, J. A., Bull. Biol. Méd. Exp., 2, 242-244 (1936).

\section{POSITION OF ADULT TESTES IN DROSOPHILA MELANOGASTER MEIGEN*}

\section{By Albert Miller}

Department of Genetics, Carnegie Institution of Washington, Cold Spring HARBOR, N. Y.

\section{Communicated December 12, 1940}

In Drosophila the effects of irradiation on genetic constitution can be readily investigated by irradiating mature sperm while it is still within the body of the male and then analyzing whatever chromosomal or genic alterations become apparent in the progeny of the treated flies. In order to reach the sperm, the rays must penetrate the intervening tissues of the fly. When rays of weak penetrating power (soft x-rays, ultraviolet rays, protons, alpha particles, cathode rays) are to be used, it becomes particularly desirable to know the position of the sperm-containing organs relative to the surface of the body and thereby to determine how maximal exposure to effective radiation may be attained. To meet this need, a study has been made of the natural position of the testes and of the effect of external pressure on their arrangement, in wild type (Swedish- $b$ ) adult males of $D$. melanogaster: The technique employed included dissection of living and fixed material, direct observation on intact specimens and study of serial sections. Fricke and Demerec ${ }^{1}$ have found that flies that have had their abdomens flattened to as little as $0.15 \mathrm{~mm}$. during irradiation may recover and function normally. To study the effect of such treatment on the position of the testes, the abdomens of etherized flies were pressed between a slide and cover glass, which were separated by another cover glass, $0.12 \mathrm{~mm}$. in thickness, to limit the degree of flattening. These flies were studied alive or were fixed while compressed and then dispected. Details of the testes are rendered discernible through the body wall of flattened flies by the addition of glycerin, but this soon kills the specimen.

Morphology and Normal Position.-The internal reproductive system of the male (Fig. 1) consists of paired testes, seminal vesicles, vasa deferentia (vasa efferentia of other authors) and accessory glands, and an unpaired ejaculatory duct with an appended ejaculatory bulb ("sperm pump"). The ejaculatory duct consists of two portions: a dilated, thick-walled anterior portion (vas deferens of other authors) extending from the vasa 